\title{
Conductividad hidráulica de una arena limosa obtenida a partir de la curva característica
}

\author{
Hydraulic Conductivity of a Silty Sand \\ Obtained from the Soil Water Characteristic Curve
}

\author{
Gallegos-Fonseca G. \\ Facultad de Ingeniería \\ Universidad Autónoma de Querétaro \\ E-mail:gfonseca@uaslp.mx \\ Leal-Vaca J.C. \\ Facultad de Ingeniería \\ Universidad Autónoma de Querétaro \\ E-mail:jclealv@hotmail.com
}

\author{
Rojas-González E. \\ Facultad de Ingeniería \\ Universidad Autónoma de Querétaro \\ E-mail:erg@uaq.mx
}

Información del artículo: recibido: junio de 2009, aceptado: octubre de 2010

\section{Resumen}

En este trabajo se presenta la determinación de la conductividad hidráulica de un suelo no saturado del tipo SM, según el criterio SUCS. Para este propósito se determinó la curva característica del suelo en trayectoria de secado y humedecimiento. Posteriormente, se ajustó dicha curva mediante el modelo de Fredlund y Xing para finalmente determinar la curva de conductividad hidráulica del suelo en trayectoria de secado y humedecimiento.

\section{Abstract}

This work shows the determination of the hydraulic conductivity of a silty sand (SM), according to USCS. For this purpose, the soil water characteristic curve at drying and wetting was first determined. Then, these curves were adjusted using the Fredlund and Xing model and finally the hydraulic conductivity of the soil for both paths was obtained.

\section{Descriptores}

- flujo de agua

- conductividad hidráulica

- curva característica

- contenido volumétrico de agua

- succión

\section{Keywords}

- water flow

- hydraulic conductivity

- soil water characteristic curve

- volumetric water content

- suction 


\section{Introducción}

Diversos problemas geotécnicos relacionados con el flujo de agua requieren la determinación del coeficiente de permeabilidad k del suelo. Algunos de estos problemas son: la estabilidad de taludes ante el humedecimiento provocado por las lluvias, diseño de presas y canales, así como muros de contención expuestos al humedecimiento y cimentaciones en general.

Se sabe que el flujo de agua en un suelo saturado depende de su granulometría, de las características del agua de poro, de la temperatura, así como de la forma, conexión y distribución del tamaño de sus poros (DTP) (Chakraborty et al., 2006); (Rojas, 2006); los poros saturados son los que contribuyen en mayor medida al flujo de agua, debido a que el gradiente de carga hidráulica, que es el principal potencial de conducción en medios saturados, actúa directamente sobre la fase líquida. En los poros que no se encuentran saturados se presenta una cierta resistencia al flujo.

El coeficiente de permeabilidad se puede determinar haciendo uso de técnicas directas e indirectas, como lo señala Juárez et al. (1992) y Whitlow (1999). Dentro de las técnicas directas se encuentran los ensayes de laboratorio mediante el permeámetro de carga variable o de carga constante, así como los ensayes in situ mediante pozos de absorción utilizando las técnicas Lugeon o Lefranc. Sin embargo, estos procedimientos sólo se aplican a medios saturados. No obstante, en numerosas situaciones el suelo no se encuentra en estas condiciones, particularmente en aquellas zonas de climas áridos y semiáridos por lo que las técnicas indirectas para hacer mediciones de estos suelos en estado no saturado cobra especial importancia; especialmente porque la medición de la permeabilidad de estos materiales toman varios meses para su realización.

Las variaciones entre la permeabilidad teórica obtenida por métodos indirectos y la experimental están ampliamente reportadas en la literatura, según lo señala Chakraborty et al. (2006). Estas variaciones emergen de las diferencias en la DTP, la densidad, la composición mineralógica, las propiedades estructurales y la presencia de materia orgánica en el suelo al momento de recolectar las muestras, así como otras características físicas y químicas, incluso en suelos de la misma textura.

Durante la realización de los trabajos de ingeniería, en ocasiones resulta muy complicado realizar el ensaye de permeabilidad in situ y por otro lado, el obtener muestras inalteradas para este propósito a veces resulta muy complicado. Para casos como estos se sugiere obtener el coeficiente de permeabilidad $k$ de forma directa como lo refiere Hillel (1982). Para este propósito es indispensable conocer tanto la presión del agua de poro como el contenido de agua del material, esta información es posible inferirla si se cuenta con la curva característica del suelo también llamada curva de retención.

Según señala Assouline (2000), la mayoría de los estudios que describen el movimiento de agua a través de medios porosos parcialmente saturados están basados en la ecuación de Richards, por lo que para resolver esta ecuación es necesaria la curva de retención del suelo.

El método que se propone utilizar para determinar el coeficiente de permeabilidad $k$ del agua en un suelo areno limoso en estado no saturado se apoya en la curva característica de este suelo en trayectoria de secado y humedecimiento, estas curvas se obtuvieron mediante la técnica de papel filtro, ASTM D 5298-03.

La curva característica se determinó mediante mediciones realizadas en el laboratorio, posteriormente se utilizó una función para ajustar cada uno de los datos obtenidos en forma experimental, como lo sugiere Assouline (2000). En la literatura de los suelos existe una gran cantidad de modelos de ajuste para este propósito; los modelos de Brooks and Corey (1964) y Van-Genuchten (1980), se encuentran entre los más populares.

Investigaciones recientes han mostrado que la curva característica se puede utilizar para determinar varios parámetros de los suelos no saturados, según Leong et al. (1997). En muchas de estas aplicaciones es obvio que la ecuación de la curva característica que mejor la describe puede simplificar la determinación de los parámetros.

La curva característica se expresa en contenido volumétrico de agua contra succión. Así, el coeficiente de permeabilidad $k$ se puede calcular en función del contenido volumétrico de agua.

\section{Concepto de la permeabilidad hidráulica del suelo}

Se han utilizado varios conceptos para explicar el flujo del agua a través de los suelos no saturados, tal es el caso de los gradientes de contenido de humedad, gradientes de succión mátrica o gradientes de carga hidráulica, por concentraciones químicas, gradientes termales y gradientes eléctricos. Todos estos gradientes son considerados otenciales de conducción como lo refiere Fredlund et al. (1993), en donde se asume que el flujo de agua va de un punto de contenido de humedad elevado a un punto de contenido de humedad bajo. Este tipo de ley de flujo no aplica totalmente cuando hay variaciones en las características del suelo encontrado. 
El flujo de agua se puede definir apropiadamente en términos de un gradiente de carga hidráulica para cada una de las fases. Por tanto, la succión mátrica es el potencial de conducción fundamental para que se presente el flujo de agua en un suelo no saturado como lo mencionan Fredlund et al. (1993). En este caso especial, en donde el gradiente de presión del aire es cero, el gradiente de succión mátrica es numéricamente igual al gradiente de presión en el agua. Esta es la situación más común en la naturaleza.

El flujo de agua a través del suelo no está gobernado únicamente por el gradiente de presión, sino también por gradientes, debidos a diferentes variaciones. Los gradientes de presión y elevación se combinan para dar el gradiente de carga hidráulica, que es el principal potencial de conductividad Fredlund et al. (1993). Los gradientes de carga hidráulica en una fase de fluido específico son el potencial de conducción para el fluido de esa fase y se presenta de igual modo para suelos saturados y no saturados, cuando los otros gradientes no son significativos.

\section{Materiales y métodos}

Con el propósito de analizar el comportamiento mecánico e hidráulico que experimenta un suelo sin potencial osmótico y bajo diferentes magnitudes de succión mátrica, es necesario emplear un material libre de sales. Por ello, se eligió una arena limosa, cuyas características se muestran en la tabla 1. La distribución de frecuencia de los tamaños de partículas se presenta en la figura 1. En esta figura se puede observar que las partículas sólidas presentan una distribución aproximadamente normal con un diámetro medio de $221.31 \mu \mathrm{m}$ y una desviación estándar de $492.27 \mu \mathrm{m}$. Físicamente el suelo presenta el aspecto mostrado en la figura 2, en la que se observa que está formado por fragmentos angulosos y subangulosos de roca ígnea con tamaño máximo de $2 \mathrm{~mm}$, también se localizaron fragmentos de cuarzo de origen ígneo, sedimentario y metamórfico; así como fragmentos aislados de feldespato potásico y plagioclasas. Además las partículas se muestran con un grado de alteración y oxidación moderado.

Con el propósito de conocer el comportamiento mecánico de este suelo bajo diversos valores de succión, se determinó su resistencia al esfuerzo cortante mediante el ensaye triaxial consolidado drenado (CD) en trayectoria de secado y humedecimiento. Para ello, se preparó un lote de 52 probetas de $3.60 \mathrm{~cm}$ de diámetro y $7.60 \mathrm{~cm}$ de altura, compactadas estáticamente para obtener un peso volumétrico máximo seco $\gamma_{\mathrm{d}}=14.88 \mathrm{kN} / \mathrm{m}^{3}$ con una humedad óptima $\omega_{\mathrm{op}}=21 \%$, determinado a partir del ensaye proctor estándar, de acuerdo con ASTM D $698-00 a^{\mathrm{e}}$.

\begin{tabular}{cccccccc}
\hline \% Grava & $\%$ Arena & \% Limo & \% Arcilla & $\%$ LL & $\%$ LP & $\%$ IP & SUCS \\
0.00 & 68.82 & 29.41 & 1.78 & 27.82 & 24.12 & 3.70 & SM \\
\hline
\end{tabular}

Tabla 1. Características del suelo ensayado

En donde LL.- Límite Líquido del suelo, LP.- Límite Plástico del suelo, IP.- Índice Plástico del Suelo y S.U.C.S.- Sistema Unificado de Clasificación de Suelos

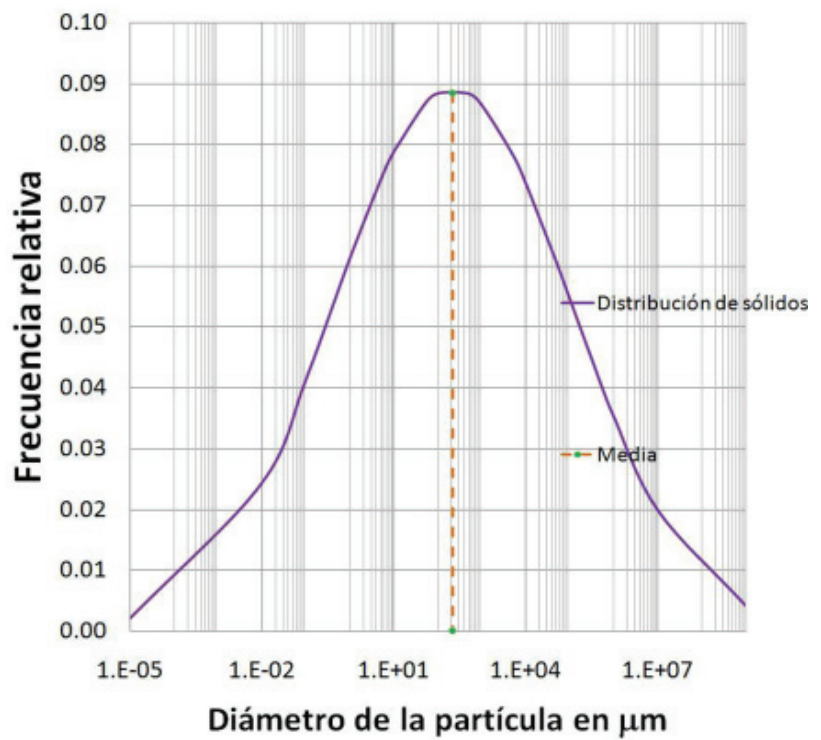

Figura 1. Distribución de frecuencia de las partículas del suelo

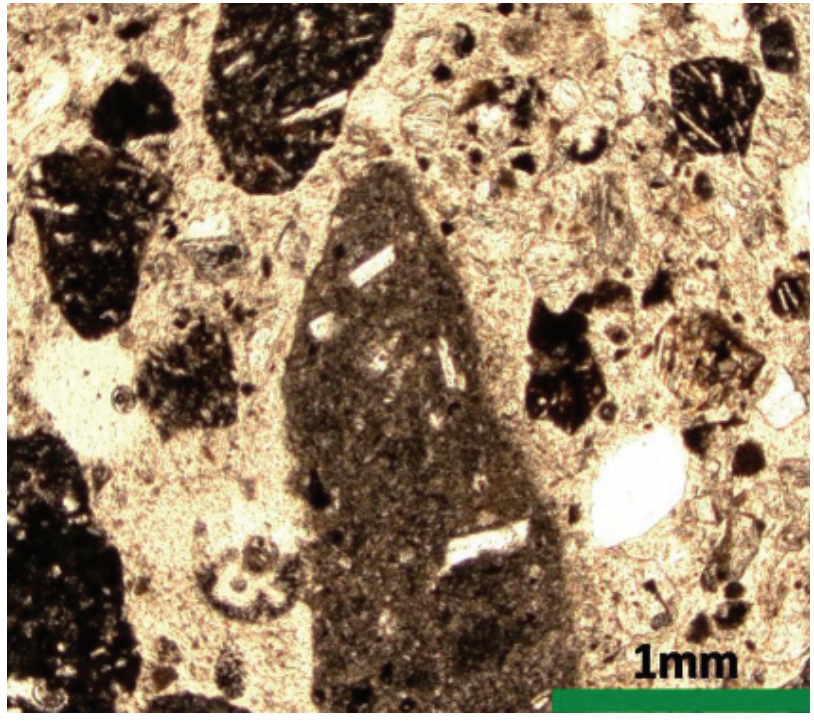

Figura 2. Arena limosa utilizada en la investigación 
Todas las probetas se fabricaron en 5 capas. En cada capa se colocó 30.50 gr de suelo, el cual se compactó con una prensa hidráulica hasta alcanzar el peso volumétrico máximo seco señalado. Entre cada una de las capas se tuvo la precaución de escarificar su superficie antes de agregar la siguiente capa y así lograr homogeneidad en las probetas.

A la mitad de las probetas se les incrementó la magnitud de la succión por medio de un secado parcial. Mientras que a la otra mitad de las probetas se les dejó secar por completo en un horno con temperatura de $105^{\circ} \mathrm{C}$ durante 24 horas. A partir de allí se inició un proceso de humedecimiento por medio de un aspersor hasta alcanzar los valores de succión previamente fijados. Los distintos valores de succión se seleccionaron buscando que quedaran distribuidos en forma equiespaciada entre el valor de succión mínima (suelo saturado) y la succión máxima (suelo seco) en la trayectoria de secado y en forma contraria para la trayectoria de humedecimiento.

Cuando los especímenes en trayectoria de secado y humedecimiento alcanzaron las condiciones previamente establecidas se les colocó dentro de una celda triaxial con succión controlada. La succión se controló por medio de la circulación de vapor de agua con una humedad relativa constante, a través de la muestra. La circulación del vapor la realizó una bomba peristáltica que trabaja a presión atmosférica y la humedad relativa se generó por medio de un recipiente que contenía una solución acuosa con cierta concentración de sales, hasta que cada una de las probetas alcanzara el equilibrio en

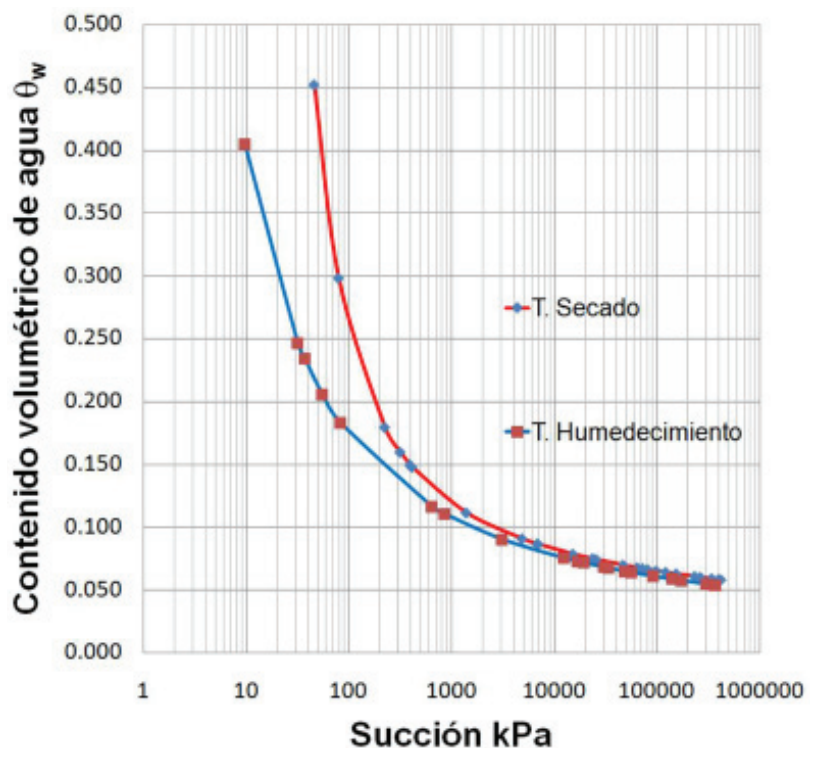

Figura 3. Curva característica del suelo en trayectoria de secado y humedecimiento la succión. Después se les aplicó un esfuerzo de confinamiento de $150 \mathrm{kPa}$ durante $24 \mathrm{hrs}$.

A cada uno de los especímenes se le determinó sus relaciones fundamentales, antes y después del ensaye triaxial, con las cuales fue posible conocer su contenido volumétrico de agua. Enseguida se les aplicó el esfuerzo desviador hasta alcanzar la falla. Posteriormente, se determinó el valor de la succión de cada muestra al final del ensaye mediante la técnica del papel filtro, ASTM D 5298-03, así como su contenido volumétrico de agua. Con esta información fue posible construir las curvas de retención que se muestran en la figura 3.

\section{Determinación de la conductividad hidráulica}

A partir de la curva característica del suelo de la figura 3 se obtuvo la curva de permeabilidad. Para lograrlo, la curva característica se dividió en $\mathrm{m}$ intervalos iguales, del contenido volumétrico de agua. En el presente caso la curva se dividió en 15 intervalos, con sus respectivos puntos medios, de cada punto medio se determinó su contenido volumétrico de agua y su correspondiente succión, procedimiento que señala Fredlund (1993).

La función de permeabilidad $k$ se obtiene mediante la ecuación 1 :

$k_{w}\left(\theta_{w}\right)=\frac{K_{s}}{K_{s c}} A_{d} \sum_{j=i}^{m}\left\{(2 j+1-2 i)\left(u_{a}-u_{w}\right)_{j}^{-2}\right\}$

Aquí, $i=1,2, \ldots . ., \mathrm{m}$

en donde:

$k_{w}\left(\theta_{w}\right)$ es el coeficiente de permeabilidad del agua predicho en $(\mathrm{m} / \mathrm{s})$,

$i \quad$ es el número de intervalo el cual aumenta con forme el contenido volumétrico de agua decrece,

$j \quad$ es el número contador de i a $m$,

$m$ es el número total de intervalos entre el conte nido volumétrico de agua saturado $\theta_{s} \mathrm{y}$ el más bajo contenido volumétrico de agua en la curva característica $\theta_{L}$

$k_{s} \quad$ es el coeficiente de permeabilidad saturado,

$k_{s c} \quad$ es el coeficiente de permeabilidad saturado teórico $(\mathrm{m} / \mathrm{s})$,

$A_{d} \quad$ es la constante de ajuste, la cual se obtiene mediante la ecuación 2.

$A_{d}=\frac{T_{S}^{2} \gamma_{w} g}{2 \mu_{w}} \frac{\theta_{S}^{p}}{N^{2}}\left(\frac{m}{s e g} k P a^{2}\right)$ 
en la que:

\begin{tabular}{|c|c|}
\hline & \\
\hline & 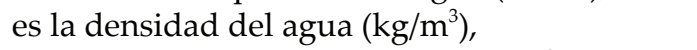 \\
\hline & es la \\
\hline & $\left(\mathrm{m}^{2}\right)$ \\
\hline & ntenido volumétrico de agua en la satu- \\
\hline & $\begin{array}{l}\text { onstante que cuenta para la interacción } \\
\text { os de varios tamaños (igual a 2), }\end{array}$ \\
\hline & $\begin{array}{l}\text { lados en- } \\
\text { aturado, }\end{array}$ \\
\hline & $\begin{array}{l}\text { es la succión mátrica correspondiente al } j \text {-ési- } \\
\text { mo intervalo en }(\mathrm{kPa})\end{array}$ \\
\hline & $\begin{array}{l}\text { resión del aire de poro y } \\
\text { el agua de poro. }\end{array}$ \\
\hline
\end{tabular}

El cálculo del coeficiente de permeabilidad, $\mathrm{k}_{\mathrm{w}}$ para un contenido de agua volumétrico específico, $\left(\theta_{w}\right)_{i}$ involucra la sumatoria de los valores de succión mátrica que corresponde al contenido de agua volumétrico en $y$ por debajo de $\left(\theta_{w}\right)_{i}$.

Este procedimiento es más recomendable para suelos arenosos que presentan una estrecha distribución de tamaño de poros Nielsen et al. (1972), debido a que los valores de la permeabilidad teóricos en estos suelos son semejantes a los resultados experimentales. La forma de la función de permeabilidad es determinada por medio de los términos que aparecen dentro de la sumatoria, así como su signo. Sin embargo, la magnitud de la función de permeabilidad necesita ajustarse con respecto al coeficiente de permeabilidad medida $k_{s^{\prime}}$ mediante el uso del factor de correlación. Por tanto, si el coeficiente de permeabilidad es medido, la función de conductividad puede obtenerse a partir de la curva característica del suelo. En la figura 4 se aprecian las curvas de con-

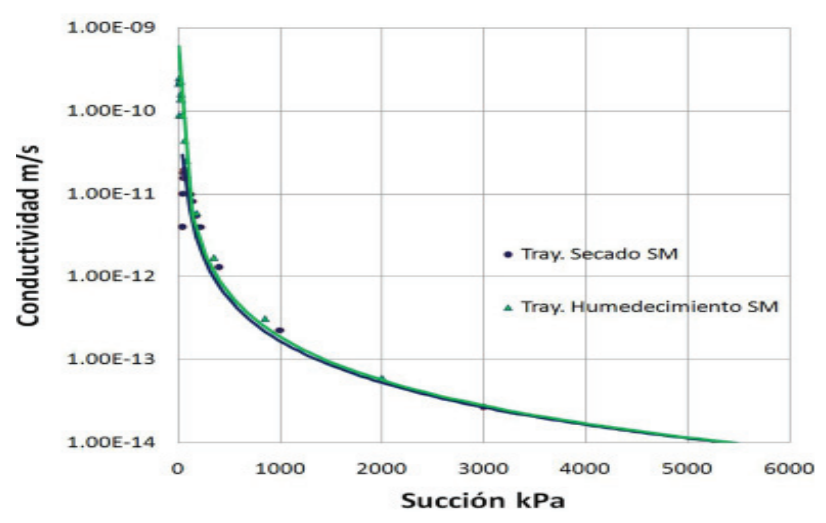

Figura 4. Curva de conductividad hidráulica del suelo en trayectoria de secado y humedecimiento ductividad hidráulica en trayectorias de secado y humedecimiento obtenidas teóricamente.

En esta investigación el coeficiente de permeabilidad saturado se determinó a partir de un ensaye de consolidación en estado saturado practicado a un espécimen de este suelo obteniéndose un valor de $k_{s}=1.44 \mathrm{E}-10 \mathrm{~m} / \mathrm{seg}$.

El contenido de agua volumétrico queda definido como $\theta_{w}=\frac{V_{w}}{V_{m}}$; donde $\theta_{w}$ es el contenido volumétrico de agua, $V_{w}$ es el volumen del agua y $V_{m}$ es el volumen de la muestra.

\section{Discusión de los resultados}

Debido a que la finalidad de este trabajo es el de exponer un procedimiento por medio del cual se puede determinar la conductividad hidráulica de un suelo no saturado, bajo distintos grados de saturación, es necesario en primer lugar contar con la curva característica del suelo, después utilizar algún modelo como el de Fredlund et al. (1994) para realizarle a los datos experimentales de la curva característica los ajustes pertinentes y posteriormente seguir el procedimiento aquí presentado. Por lo anterior, si se requiere determinar la conductividad hidráulica en ambas trayectorias, es necesario también poder contar con la función de la curva característica en ambas trayectorias. Según lo refiere Leong et al. (1997) esto permitirá estimar la conductividad hidráulica de cualquier suelo de forma rutinaria y aplicarlo en la solución de problemas geotécnicos que involucren el flujo de agua en suelos no saturados.

Ya que al obtener las funciones de conductividad hidráulica se toma en cuenta la textura, peso específico y materia orgánica presente en el suelo, a través de la función de la curva característica del suelo (Arya et al., 1999), la conductividad hidráulica así obtenida se encuentra determinada por los parámetros que describen la estructura de los poros del suelo. En la figura 4 se puede apreciar cómo en la medida en la que el suelo experimenta menor succión, la facilidad con la que el agua pasa a través del suelo se acrecienta; sin embargo, para magnitudes de succión elevada la facilidad con la que el agua fluye decrece significativamente, en ambas trayectorias.

Por otro lado, también se puede apreciar que un suelo alcanza mayor velocidad de flujo cuando se trata de un proceso de humedecimiento, mientras que en un proceso de secado la velocidad alcanzada es inferior. Además, cuando el suelo se encuentra experimentando la máxima succión, el comportamiento de ambas curvas de conductividad alcanza una tendencia asintótica con respecto al eje horizontal. 


\section{Conclusiones}

En la solución de problemas geotécnicos que requieran determinar los cambios de volumen o flujo del suelo es necesario tener presente que:

La curva característica de un suelo es una propiedad exclusiva de la distribución del tamaño de poro del material, la cual obedece a un acomodo particular de los sólidos.

Obtener la curva característica del suelo mediante la técnica del papel filtro es un procedimiento sencillo y económico.

Es necesario contar con la función matemática que describe la curva característica del suelo para determinar su curva de conductividad hidráulica.

La curva de conductividad es una valiosa herramienta que permite conocer el comportamiento hidráulico de un suelo no saturado.

El procedimiento para obtener la conductividad hidráulica del suelo mediante el método de la curva característica resulta ser sencillo y rápido.

\section{Referencias}

Annual book of ASTM STANDARDS. International Standard World Wide. Section four Construction Volume 04.08. Soil and Rock (I). D420-D-5611. 2004.

Assouline S., Tartakovsky D.M. Unsaturated Hydraulic Conductivity Function Based on a Soil Fragmentation Process. Department of Environmental Physics, Institute of Soil, Water and Environmental Sciences, Volcanic Center. Agricultural Research Organization, Bet Dagan, Israel. Mathematical Modeling and Analysis Group, Los Alamos National Laboratory, Los Alamos, New Mexico. 2000.

Bishop A.W. The Principle of Effective Stress. Teknisk Ukeblad, 39:859-863. 1959.
Brookcs R.H., Corey A.T. Hydraulic Properties of Porous Media. Colorado State Univ. Hidrol. Paper. (3):27. Mar 1964.

Chakraborty D., Chakraborty A., Santra P., Tomar R.K., Garg R.N., Sahoo R.N., Ghosal Choudhury S., Bhavanarayana M., Kalra N. Prediction of Hydraulic Conductivity of Soils from Particle-Size Distribution. 1 Division of Agricultural Physics. En: Indian Agricultural Research Current Science, Vol. 90, No. 11, 10 June 2006. Research Comunications.

Fredlund D., Rahardjo H. Soil Mechanical for Unsaturated Soils. I Edición. Ney York. A Wiley-Intescience Publications, Inc. 1993.

Fredlund D.G., Xing A. Equation for the Soil Water Characteristic Curve. Canadian Geotechnical Journal, 31(3): 521-532, 1994.

Hillel D. Introduction to Soil Physics. Academic Press. New York. 1982.

Juárez-Badillo E. y Rico-Rodríguez A. Mecánica de suelos. Tomo 1 y 2. XIV Edición. México. Limusa.1992.

Lalit M.A., Feike J.L., Van-Genuchten M.Th., Shouse P.J. Scaling Parameter to Predict the Soil Water Characteristic from ParticleSize Distribution Data. USDA-ARS, US. Salinity Lab., 450 W. Big Springs Rd., Riverside, CA 92507. Published in Soil Sci. Soc. Am. J. 63:510-519. 1999.

Leong E.C., Rahardjo H. Review of Soil Water Characteristic Curve Equations. Journal of Geotechnical and Geoenvironmental Engineering, 123(12). December 1997.

Nielsen D.R., Jackson R.D., Cary J.W., Evans D.D. Soil Water. Amer. Soc. Agronomy and Soil Sci. Amer., Madison. WI, 1972.

Rojas E., Rojas F. A Probabilistic Model for the Soil-Water Characteristic Curve. Proceeding of IV International Congress of Unsaturated Soils in Arizona. 2006.

Van-Genuchten M.Th. A Closed-Form Equation for Predicting the Hydraulic Conductivity of Unsaturated Soils. Soil Sci Am J, 44:892-898. 1980.

Whitlow R. Fundamentos de mecánica de suelos. II Edición. México. Compañía Editorial Continental S.A. de C.V. 1999.

\section{Semblanza de los autores}

Gustavo Gallegos-Fonseca. Es ingeniero civil. Maestro en ciencias en la línea de mecánica de suelos, alumno del doctorado en ingeniería de la Facultad de Ingeniería de la Universidad Autónoma de Querétaro. Es profesor de la licenciatura en ingeniería civil de la UASLP ZM desde 1993 a la fecha.

Julio Leal-Vaca. Es ingeniero civil. Maestro en ciencias en la línea de mecánica de suelos, actualmente es alumno del doctorado en ingeniería de la Facultad de Ingeniería de la Universidad Autónoma de Querétaro. Es profesor de la licenciatura en ingeniería civil de la U de $\mathrm{G}$ desde 1998 a la fecha.

Eduardo Rojas-González. Actualmente es profesor investigador de la Facultad de Ingeniería de la Universidad Autónoma de Querétaro. 